\title{
Development of Computer Education Program Using LOGO Programming and Fractals Learning for Enhancing Creativity: Focus on Creative Problem-Solving
}

\author{
Namje Park \\ Department of Computer Education, Teachers College, Jeju National University \\ namjepark@jejunu.ac.kr
}

\begin{abstract}
The paper was aimed to investigate the effect of the LOGO programing language on the creativity development of elementary school students. And, this paper analyzed the statuses of STEAM education. And, suggested factors to realize successful STEAM education materials. An examination of the research on LOGO indicates that research is being amply conducted overseas on LOGO and thinking ability, planning ability and programming, the cognitive effects on programming, and the effects of learning LOGO on cognitive styles.
\end{abstract}

Keywords: Computer Education, LOGO, EPL, Creative Problem-Solving, Creativity

\section{Introduction}

Such computational sciences and IT education can also be found in the concept of STEAM education. Yakman defined the boundaries of Science(S), Technology(T), Engineering(E), Arts(A), and Mathematics(M) in STEAM education. He suggested that the contents of computational sciences belong to the Computer in Engineering (E) field and the contents of IT belong to informational technology under Technology(T). The STEAM education using IT is an attractive educational method for the digital generation students to easily and pleasantly learn the contents of mathematics, sciences, and technology. The educational methods that integrate IT and other fields of academia have been attempted before STEAM education. In addition, the reason for a brighter footlight for IT as the transition from STEM education to STEAM education occurs is that it occupies a large section in the field of Art in the digital environment.

The paper was aimed to investigate the effect of the LOGO programming language on the creativity development of elementary school students. LOGO was created at the MIT Artificial Intelligence Laboratory, the United States, by Seymour Papert and his colleagues in the late 1960s. Heavily influenced by a cognitive psychologist, Piaget, Papert pointed out that the school education not just failed to satisfy student's curiosity, but also discouraged children's innate intellectual curiosity. He developed LOGO from the perspective of constructivism to satisfy the children's inborn desire. An examination of the research on LOGO indicates that research is being amply conducted overseas on LOGO and thinking ability, planning ability and programming, the cognitive effects on programming, and the effects of learning LOGO on cognitive styles. However, few are found in the domestic elementary schools. One reason is that BASIC was mainly taught as part of programming learning in the early years of computer education rather than learning for LOGO from the curriculum.

\section{Creative Problem-Solving and STEAM Education}

Guilford (1959) suggested that the human thoughts are divided into convergent and divergent thoughts and these are comprised of the fluency that symbolizes the speed of 
thoughts, adaptability that symbolizes the width of thoughts, uniqueness that symbolizes the newness of thoughts, accuracy that symbolizes the areas not seen by others, sensitivity that symbolizes the response to objects, and redefinition and recreation. Arieti (1976) suggests memory, recognition, evaluation, convergent thoughts, and divergent thoughts as the intelligent factors of creative thinking. Firestein and Treffinger (1983) emphasized the importance of divergent thoughts as the components of creativity and also suggested the abilities to marvel, value, transition, and concentrate. The creative problem-solving does not have only one way out, but many and is a process to find the optimal solution among many alternatives.

As the low interests and accomplishments of American teenagers in math and science, the STEM (science, technology, engineering, mathematics) education started as an educational solution. However, the STEM education was missing a very important piece. This is that Art, a comparatively competitive and innovative field as STEM in creativity, was also needed. In addition, the science education could not keep up with the current changes in science, technology, and engineering and the teenagers who are used to the various advanced technology products were bound to lose interests as well as creating a gap in creativity cultivation in science education during elementary and middle school years. Therefore, the experts argued for "amicability between science and art" because a dichotomous thought that art is illogical and science is not creative ruined the future and the art and science should be taught together before the concept of STEAM education emerged. In this perspective, the art education is crucial in developing creativity that is highly valued in modern education; therefore, the art education should be added to the education of science, technology, engineering, and mathematics.

\section{LOGO Programming and FRACTALS based on CPS Model}

\subsection{Linking Computer Education to Elementary School Mathematics Courses}

The ultimate goal of elementary school computer education is using computer education to enhance creativity, problem solving ability, and logical thinking. The same goal applies to mathematics education. A lot of the learning experiences present in computer education can help students achieve the goals of their mathematics education, and vice versa. Therefore, linking elementary school mathematics education to elementary school computer education can produce powerful synergy.

The five sections of elementary mathematics education are numbers and calculations, figures, measurements, probability and statistics, and finally regulation and problem solving ability.

\subsection{Methods of Applying LOGO to Elementary School Computer Education}

We drew up lesson plans for a 4-hour computer education course that used LOGO and fractal theory. The goal was focused not on studying the basic functions of programming but on getting the maximal effect from applying the minimum of functions. The lesson plan is shown in figure 1. In the first lesson, we studied basic LOGO commands and the concept of fractals. In the second lesson, we made and studied Basic-T fractals using LOGO. In the third lesson, we made T fractals which were more difficult than Basic-Y fractals. In the last lesson, we made fractal procedures using figures which were covered in the first to third lessons and that the students had learned and in their own way. The overall flow of study was composed with basic and intensified process for graded lesson. 


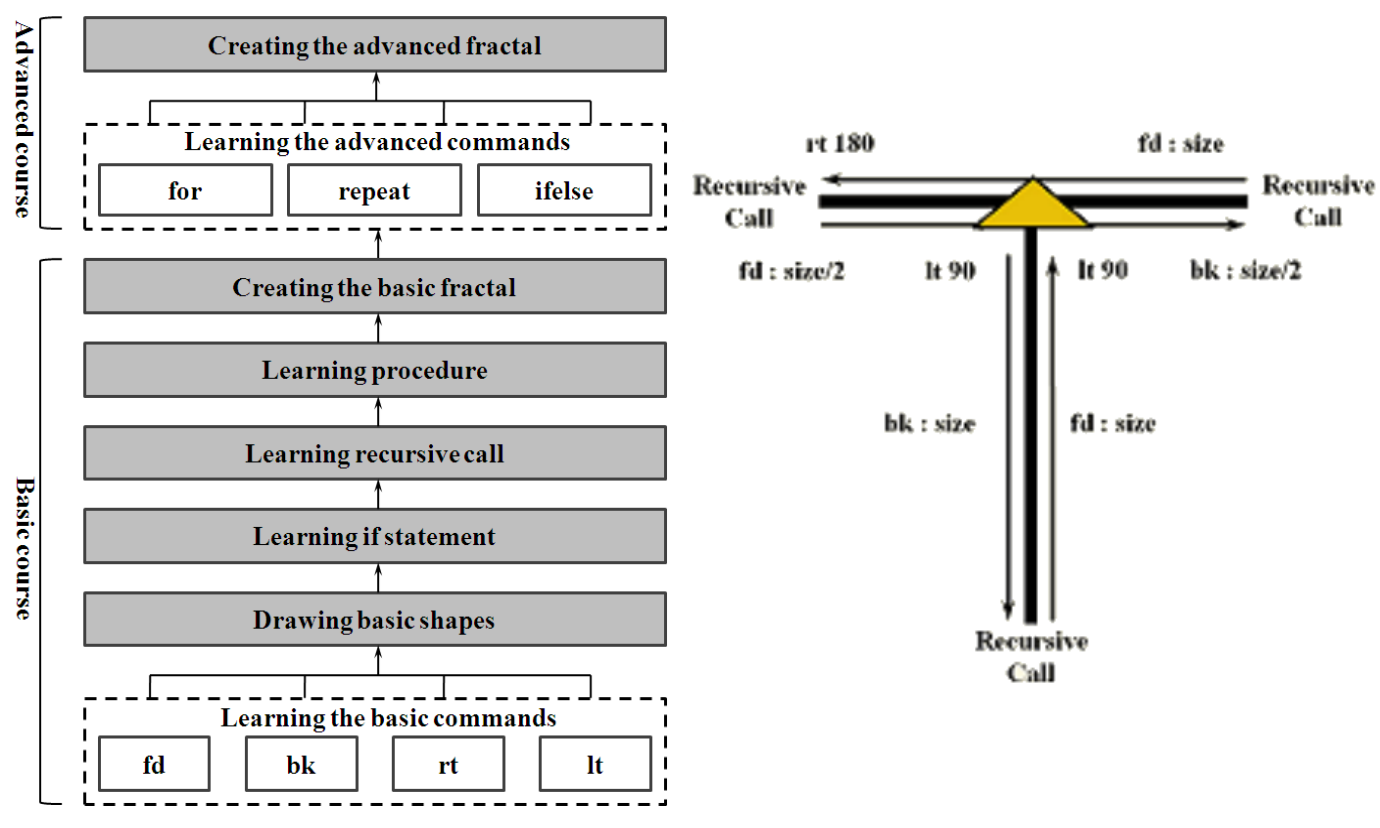

Figure 1. Fractal Geometry Screen Developed by LOGO (T Fractal)

First, the level of interest of the students has to be taken into consideration. It is possible that students will lose interest when the teacher teaches basic commands, and accordingly, the teacher has to minimize the presentation of functions. Also, the teacher can increase student interest by providing various applications which are suitable for the level of the students. Secondly, the teacher has to emphasize procedures and recursive calls because they are crucial to making fractals. Third, when the teacher teaches the Y fractal, intervention has to be minimized to offer plenty of opportunities because it applies what was learned about Basic-T fractals. Lastly, advanced lessons should be given to students who get good results, and remedial education should be given to the other students.

\section{Experiment and Verification of Validity}

The satisfaction for the class is as shown in figure 2 when three types of education are subsequently conducted three times. At first, there is not much difference in academic satisfaction as there was not much difference in input values. However, as the experiment continues, LOGO leaning model, which is conducted subsequently three times showed more satisfaction in class. This may be caused by the followings: First, the learning model using the LOGO programming could get more attention from students compared to the general mathematical education model as it reflects more interesting factors in the learning model. Second, it also helped to conduct the class in more various ways of educations and expressions and thus attracted more attention from students, causing the rise in academic satisfaction.

Third, in the process of selection among three different education models, the teacher has more chance of selecting the effective learning model and thus selectively reducing the potential conflict. 


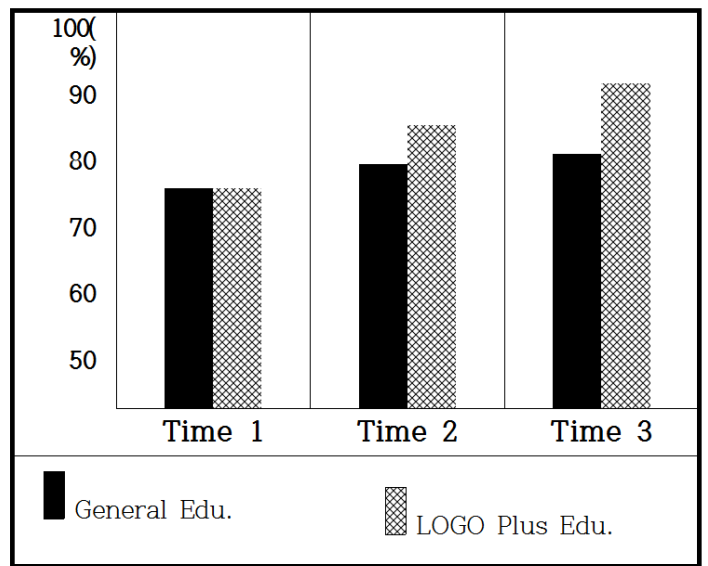

Figure 2. Result of Class Satisfaction (LOGO Programming Education).

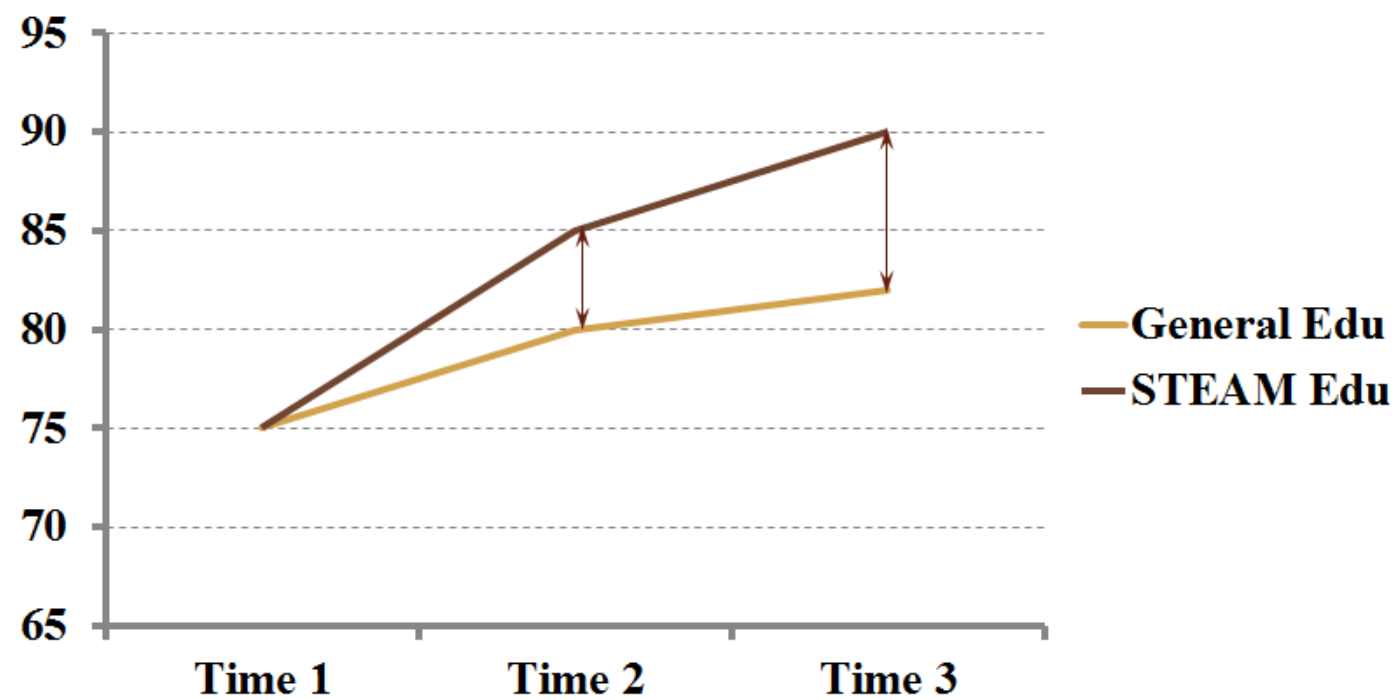

Figure 3. Result of Class Satisfaction (STEAM Education).

As the experiment continues, STEAM learning model, which is subsequently three times showed more satisfaction in class. Lately, plenty of education programs have been provided for equal education of those who are subjected to social and economic inequality. However, it is doubtable whether these education programs are appropriate to the objective of each curriculum, and whether the importance is recognized. An experiment conducted by this study showed a significant difference just in 1-2 items of areas for selfconfidence, curiosity and openness except for tolerance area that is one of the creative personality areas. Judging from the creative personality standard used in the study, the effectiveness of educational programming class on the training of creative personality is not clear, but the difficulty in quantifying and measuring the creativity and the existence of innumerable individual and environmental variables make it difficult to determine its effectiveness. Nevertheless, it is necessary to improve the quality of the class through diverse ways such as improving teaching methods and the curriculum to foster versatile creative personality of the students, such as self-confidence, curiosity, openness, and adventurous spirit through using educational programming class. In addition, it is expected to be upgraded the quality of the class that is offered to elementary students and help the students cultivate their creative personality as well. 
The significant results of the paper can be summarized as follows. First, the learning activities of LOGO programming language are shown to have effects on the development of creativity. Second, the LOGO programming language is found to influence on the enhancement of sub-items; fluency, originality, abstractness, elaboration and resistance. Third, its contribution to improvement of creativity was identical between male and female. Based on the study results, the LOGO programming language is considered to have positive effects on creativity development by stimulating brain activity of children.

The paper deals with the results of an experiment on the effects of the LOGO programming language on the development of creativity and the research is considered that the following studies should be conducted: First, the ICT curriculum for computer education should include a programming language such as LOGO. Second, training programs for teachers are required for LOGO programming language learning. Third, development of various teaching methods is necessary for LOGO programming language learning. Fourth, continuous education is important with the curriculum that doesn't end within a school year but is connected to the following school year.

Since the subjects of this paper were limited to elementary school students and just few LOGO programming classes were conducted, the tests were carried out without sufficient understanding of the LOGO programming language. Thus it can be difficult to generalize the results. Therefore, follow-up research should be conducted to learn! the LOGO programming language over long periods and to satisfy the results.

\section{Conclusions}

To realize the STEAM education, the factors on how to interrelate and integrate science, technology, engineering, art, and mathematics as well as the factors that are needed in realizing the STEAM education in creativity in addition to the considered factors in contents need to be decided, which in reality, makes the creation of STEAM materials into a system science or system engineering. In other words, the many factors need to harmonize in a creative and appropriate way along with the theoretical foundation and applications in a systematic way. This paper analyzed the statuses of STEAM education. And, suggested factors to realize successful STEAM education materials.

\section{Acknowledgments}

This work was supported by the Korea Foundation for the Advancement of Science and Creativity(KOFAC) grant funded by the Korea government(MOE). And, this work was supported by the Ministry of Education of the Republic of Korea and the National Research Foundation of Korea (NRF-2015S1A5A8018037)

\section{References}

[1] J. P. Guilford, "Traits of creativity in Creativity and its Cultivation", Harper and Row, (1959).

[2] S. A. Arieti, "Creativity - the Magic Synthesis, Basic Books", Inc. Publishers, (1976).

[3] Y. Ko, J. An and N. Park, "Development of Computer, Math, Art Convergence Education Lesson Plans Based on Smart Grid Technology", In: T. Kim, A. Stoica and W. Fang, (eds.), vol. 339, (2012), pp. 109114.

[4] N. Park and Y. Ko, "Computer Education's Teaching-Learning Methods Using Educational Programming Language Based on STEAM Education”, In: J. Park, A. Zomaya and S. Yeo, (eds.), vol. 7513, (2012), pp. 320-327.

[5] Y. Ko and N. Park, "Experiment and Verification of Teaching Fractal Geometry Concepts Using a Logo-Based Framework for Elementary School Children”, In: T. Kim, H. Adeli, D. Slezak, (eds.), vol. 7105, (2011), pp. 257-267.

[6] J. An and N. Park, "The Effect of EPL Programming Based on CPS Model for Enhancing Elementary School Students' Creativity”, In: J. J. Park, Y. Jeong and S. O. Park, (eds.), vol. 181, (2012), pp. 237244. 
[7] J. An and N. Park, "Computer Application in Elementary Education Bases on Fractal Geometry Theory Using LOGO Programming”, In: J. J. Park, H. Arabnia and H. Chang, (eds.), vol. 107, (2011), pp. 241249.

[8] N. Park, "Implementation of Terminal Middleware Platform for Mobile RFID computing", International Journal of Ad Hoc and Ubiquitous Computing, vol. 8, no. 4, (2011), pp. 205-219.

[9] Y. Kim and N. Park, "Development and Application of STEAM Teaching Model Based on the Rube Goldberg's Invention”, In: S. Yeo, Y. Pan and Y. S. Lee, (eds.), vol. 203, (2012), pp. 693-698.

[10] Y. Kim and N. Park, "The Effect of STEAM Education on Elementary School Studen's Creativity Improvement”, In: T. Kim, A. Stoica and W. Fang, (eds.), vol. 339, (2012), pp. 115-121.

[11] Y. Kim and N. Park, "Elementary Education of Creativity Improvement Using Rube Goldberg's Invention”, In: J. H. Park, J. Kim and D. Zou, (eds.), vol. 180, (2012), pp. 257-263.

[12] N. Park, J. Kwak, S. Kim, D. Won and H. Kim, "WIPI Mobile Platform with Secure Service for Mobile RFID Network Environment", In: H. T. Shen, J. Li, M. Li, J. Ni and W. Wang, (eds.), APWeb Workshops, LNCS, (2006).

[13] J. Hong and N. Park, "Teaching-learning Methodology of STS Based on Computer and CAI in Information Science Education", In: S. Yeo, Y. Pan and Y. S. Lee, (eds.), vol. 203, (2012), pp. 733-738.

[14] H. Jeon and N. Park, "Creative Problem-Solving: School Computer Education Using LOGO Programming and Fractals", International Conference on Platform Technology and Service (PlatCon-14), (2013); Jeju, Korea.

[15] B. Lee and N. Park, "Performance Improvement Based Authentication Protocol for Inter vessel Traffic Service Data Exchange Format Protocol Based on U-Navigation System in WoT Environment", Journal of applied mathematics, Hindawi Publishing Corporation, (2014).

[16] N. Park, "Development and Application of Elementary STEAM Career Education Program Using LOGO Programming and Fractals Learning", Advanced Science Letters, vol. 21, no. 3, (2015), pp. 549552.

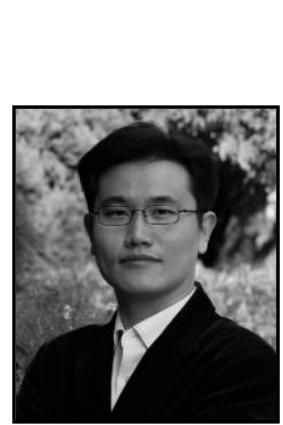

\section{Author}

Namje Park, He received the BSC degree in information industry from Dongguk University, Korea in 2000, and received his M.E., and $\mathrm{Ph} . \mathrm{D}$. degrees in Information Engineering from Sungkyunkwan University in 2003, and 2008 respectively. He is a Professor of Department of Computer Education in Teachers College at Jeju National University since 2010. He has been serving as a Research Scientist of Arizona State University since 2010. Prior to joining the researcher at ASU, he had worked as a post-doc at University of California, Los Angeles for 1 year. And he had an appointment as the senior engineer of the information security research division of the Electronics and Telecommunication Research Institute for 6 years. $\mathrm{He}$ is concerned in the information security technology field for the mobile environments, IoT system, Smart Grid, Mobile XML Security, Web Services Security, Ubiquitous computing including RFID/WSN and a variety of cryptographic technologies. He has many talks related in mobile and information security technologies, computer education. 